\title{
TOXICIDADE AGUDA DOS HERBICIDAS DIURON E HEXAZINONA À Danio rerio
}

\author{
BRUNO MOREIRADASILVA* \\ MICHELAUGUSTO CAMARGO RAVANELI** \\ CRISTINA FILOMÊNA PEREIRA ROSA PASCHOALATO***
}

\begin{abstract}
O objetivo deste trabalho foi avaliar a toxicidade de herbicida comercial aplicado extensivamente na cana-de-açúcar, composto por diuron, hexazinona e ingredientes inertes, usando-se Danio rerio como organismo-teste. Os ensaios foram realizados em laboratório e seguiram recomendações da ABNT (2004) e USEPA (2002). Os parâmetros de controle da água foram $\mathrm{pH}$, dureza total, condutividade, oxigênio dissolvido e temperatura, observando-se a atividade natatória e a mortalidade dos organismos. $\mathrm{A} \mathrm{CL}_{50}$ do herbicida comercial para Danio rerio foi de $581,50 \mathrm{mg} . \mathrm{L}^{-1}$. Em concentrações a partir de $50 \mathrm{mg} . \mathrm{L}^{-1}$ do herbicida comercial foram observadas alterações comportamentais significativas, como hipoatividade relacionada ao movimento natatório em mais de $50 \%$ dos organismos. Para avaliação da toxicidade dos ingredientes inertes, responsáveis por $40 \%(\mathrm{~m} / \mathrm{m})$ da composição do herbicida, foram testados os compostos hexazinona e diuron separadamente. Nas concentrações estudadas, a hexazinona e o Diuron não resultaram em mortalidade ou alteração ao movimento natatório. Concluiu-se que os ingredientes inertes da composição do herbicida comercial foram os principais causadores da toxicidade aguda causada ao Danio rerio.
\end{abstract}

PALAVRAS-CHAVES: CANA-DE-AÇÚCAR; ECOTOXICOLOGIA; Danio rerio; DIURON; HEXAZINONA.

* $\quad$ Aluno de iniciação científica, Curso de Engenharia Química, Universidade de Ribeirão Preto (UNAERP), Ribeirão Preto, SP (e-mail: bruno_rib@hotmail.com).

** Biólogo, Mestre em Tecnologia Ambiental pela UNAERP, Ribeirão Preto, SP (e-mail: mravaneli@hotmail.com).

*** Engenheira Química, Mestre e Doutora em Engenharia Civil (Hidráulica e Saneamento), Docente do programa de Pós-Graduação em Tecnologia Ambiental, UNAERP, Ribeirão Preto, SP (e-mail: cpaschoa@unaerp.br). 


\section{INTRODUÇÃO}

A crescente demanda nos mercados interno e externo por combustíveis renováveis, especialmente o etanol, atrai investimentos para a formação de novas áreas de cultivo da cana-deaçúcar. Aárea plantada de cana-de-açúcar no Brasil alcança, aproximadamente, 7 milhões de hectares, perfazendo $2 \%$ da terra agricultável do país (UNICA, 2008). Segundo estimativa do IEA (2006) para e safra 2015/2016, a área plantada atingirá 12,2 milhões de hectares, o suficiente para gerar cerca de 36 bilhões de litros de etanol.

No estado de São Paulo, a região de Ribeirão Preto apresenta intensa atividade agrícola com monocultivo de cana-de-açúcar sobre área de descarga e recarga do Aquífero Guarani (o mais importante de toda América Latina), que abastece na sua totalidade a população de Ribeirão Preto (PESSOA et al., 2003). Dentre os diversos agrotóxicos utilizados na cultura da cana-de-açúcar, destaca-se o herbicida constituído pela mistura de diuron e hexazinona. O Quadro 1 mostra as principais características dos compostos diuron e hexazinona.

\section{QUADRO 1 - PRINCIPAIS CARACTERÍSTICAS DOS COMPONENTES ATIVOS DIURON E HEXAZINONA, UTILIZADOS NA CULTURA DE CANA-DE-AÇÚCAR}

\begin{tabular}{|c|c|c|}
\hline Características & Hexazinona & Diuron \\
\hline $\begin{array}{l}\text { Grupo químico } \\
\mathrm{N}^{\circ} \text { CAS }\end{array}$ & $\begin{array}{c}\text { Triazona } \\
5123-04-02\end{array}$ & $\begin{array}{c}\text { Uréia } \\
330-54-1\end{array}$ \\
\hline Nome químico & 3-ciclohexil-6(dimetilamino) 1-metil- & $\begin{array}{l}\text { 3-(3,4-diclorofenil)-1,1- } \\
\text { dimetiluréia }\end{array}$ \\
\hline $\begin{array}{l}\text { Fórmula bruta } \\
\text { Massa molar }\end{array}$ & $\begin{array}{c}\mathrm{C}_{12} \mathrm{H}_{20} \mathrm{~N}_{4} \mathrm{O}_{2} \\
252,32\end{array}$ & $\begin{array}{c}\mathrm{C}_{9} \mathrm{H}_{10} \mathrm{C}_{12} \mathrm{~N}_{2} \mathrm{O} \\
233,1\end{array}$ \\
\hline Estado físico & $\begin{array}{l}\text { sólido cristalino marrom claro de } \\
\text { odor característico }\end{array}$ & $\begin{array}{c}\text { sólido cristalino esbranquiçado de } \\
\text { odor característico }\end{array}$ \\
\hline Classe & Inibidor da fotossíntese & Inibidor da fotossíntese \\
\hline Pressão de vapor a $25^{\circ} \mathrm{C}$ & $\begin{array}{c}1,9 \times 10^{-7} \mathrm{~mm} \text { de } \mathrm{Hg} \text { (volatilidade } \\
\text { baixa) }\end{array}$ & $\begin{array}{c}2,3 \times 10^{-9} \mathrm{~mm} \text { de } \mathrm{Hg} \text { (volatilidade } \\
\text { muito baixa) }\end{array}$ \\
\hline $\begin{array}{l}\text { Solubilidade em água em } \\
\mathrm{pH} 7 \text { a } 25^{\circ} \mathrm{C}\end{array}$ & 29,8 g/L (solubilidade elevada) & 42 mg/L (solubilidade baixa) \\
\hline $\begin{array}{l}\text { Meia-vida no solo sob } \\
\text { condições aeróbicas }\end{array}$ & 115 dias & 118 dias (102 a 134 dias) \\
\hline $\begin{array}{l}\text { Meia-vida (fotólise na } \\
\text { água) }\end{array}$ & $>30$ dias & 43 dias \\
\hline Classificação tóxica & Classe III & Classe III \\
\hline Uso agrícola & $\begin{array}{c}\text { Aplicação em pré e pós emergência } \\
\text { das plantas infestantes na cultura } \\
\text { de cana-de-açúcar }\end{array}$ & $\begin{array}{c}\text { Aplicação em pré e pós } \\
\text { emergência das plantas } \\
\text { infestantes na cultura de cana- } \\
\text { de-acúcar }\end{array}$ \\
\hline $\mathrm{LMR}(\mathrm{mg} / \mathrm{Kg})$ & 0,1 & 0,1 \\
\hline
\end{tabular}

Fonte: LANÇAS, 2002.

Matallo et al. (2003) estudaram a lixiviação do herbicida diuron em coluna de solo do tipo Neossolo Quartzarênico e confirmaram o transpasse do diuron. O estudo sugeriu a possibilidade de contaminação das águas subterrâneas e a necessidade de se aprofundar estudos em relação ao lençol freático e à zona não saturada em regiões com cultura de cana-de-açúcar.

Considerando a necessidade de uso dos corpos d'água como mananciais destinados ao abastecimento da população, em 2009 foram publicados estudos sobre a eficiência da tecnologia de tratamento de água do tipo convencional (coagulação, floculação, decantação, filtração e desinfecção) 
na remoção dos microcontaminantes diuron e hexazinona. Os resultados mostraram que os compostos investigados não foram removidos pela tecnologia convencional de tratamento de água. Quando ocorre a presença desses contaminantes nos mananciais utilizados para o abastecimento, a ineficiência de remoção pode causar prejuízos na qualidade da água potável a ser distribuída (DANTAS et al., 2009; PASCHOALATO et al., 2009; PÁDUA, 2009).

Politano e Pissarra (2005) avaliaram por foto-interpretação as áreas de abrangência dos diferentes estados da erosão em solos de canaviais da região de Ribeirão Preto-SP. Constataram a possibilidade de contaminação dos corpos d'água, devido à presença de erosão acelerada severa a extremamente severa, que revelaram forte impacto sobre a degradação do solo e qualidade da água.

Pessoa et al. (2007) avaliaram o potencial de transporte e lixiviação de vários agrotóxicos. Consideraram as características físico-químicas de 236 princípios ativos, dentre os quais o diuron e a hexazinona, que demonstraram capacidade de contaminação de águas subterrâneas e superficiais (rios e lagos).

A Portaria $n^{\circ} 518$, do Ministério da Saúde que define o padrão de potabilidade do Brasil não cita diuron e hexazinona (BRASIL, 2004). Na Resolução CONAMA ${ }^{\circ} 357 / 2005$, que estabelece padrões de qualidade para as águas superficiais por classes, os compostos diuron e hexazinona também não são citados. O parágrafo $4^{\circ}$, do artigo 8 dessa Resolução, recomenda que as possíveis interações entre as substâncias e a presença de contaminantes não listados, passíveis de causar danos aos seres vivos, deverão ser investigadas mediante ensaios ecotoxicológicos, toxicológicos, ou outros métodos cientificamente reconhecidos (BRASIL, 2005).

Os ensaios de toxicologia aquática destacam-se como importante ferramenta para avaliação da sensibilidade de organismos a fatores ambientais desfavoráveis como, efluentes tóxicos, poluentes físicos, químicos e medicamentos. São realizados com a finalidade de regulamentação dos limites aceitáveis de contaminação ambiental, homologação e registro de produtos químicos comerciais usados no meio ambiente e testes em medicamentos, que permitem avaliar a eficácia e também os efeitos deletérios dos compostos químicos (LOMBARDI, 2004).

A Companhia de Tecnologia de Saneamento Ambiental do Estado de São Paulo (CETESB) realiza ensaios ecotoxicológicos com organismos aquáticos para determinação dos efeitos tóxicos causados por um ou mais agentes químicos em amostras de águas, sendo tais efeitos detectados por respostas fisiológicas. Buscando-se resposta mais ampla dos efeitos tóxicos de várias substâncias, muitos estudos relacionados à ecotoxicidade estão sendo realizados com grande variedade de organismos bio-indicadores. Helfrich et al. (1996) utilizaram a truta Arco-iris; Frello (1998) o Barrigudinho, Jonsson e Maia (1998) o Lambari e Resgalla Jr. et al. (2002) a Carpa comum.

O organismo Danio rerio (peixe paulistinha), espécie de ovíparo de hábitos alimentares onívoros, apresenta grande adaptabilidade, podendo sobreviver em diferentes condições ambientais. Trata-se de organismo de fácil reprodução em ambiente artificial e facilmente encontrado em lojas de peixes ornamentais.

Dentre os estudos realizados com Danio rerio para avaliar a toxicidade de compostos químicos destaca-se o trabalho de Nakagome, Noldin e Resgalla Junior (2007) com os herbicidas oxifluorfem, oxadiazona e clomazona e os inseticidas lambdacialotrina, betaciflutrina, carbofurano e fipronil, empregados em lavouras de arroz. Ferrão Filho et al. (2007) utilizaram Danio rerio para avaliar a toxicidade de concentrações subletais de cianobactérias da espécie Cylindrospermosis raciborskii. Holth et al. (2008) usaram Danio rerio para avaliar a toxicidade dos hidrocarbonetos policíclicos aromáticos (HPA) e alquilfenois (AF).

De acordo com Baganz (2005), a redução da atividade metabólica pode representar estratégia eficaz se a exposição ao agente agressor tiver natureza temporária e se a energia economizada para a mitigação do processo tóxico for suficiente para suprir esse período. No entanto, atividade reduzida devido a longo período de exposição pode ocasionar redução dos hábitos alimentares e de cópula, possibilitando colapso metabólico no organismo. Como a natação constitui aspecto central para a biologia dos peixes, sua redução pela exposição a agentes tóxicos pode implicar na diminuição das interações interespecíficas e intra-específicas.

Justificam o presente trabalho as grandes quantidades de herbicidas (diuron e hexazinona) 
usados na monocultura de cana-de-açúcar, o crescente processo de erosão e a incapacidade de remoção desses microcontaminantes nas estações de tratamento de água, aliada à falta de citação desses compostos nos padrões de qualidade das águas no território nacional.

Neste estudo objetivou-se a determinação da concentração mínima do herbicida para ocasionar $100 \%$ da letalidade dos peixes Danio rerio, a concentração máxima que não causa mortalidade $\left(\mathrm{CE}_{\text {zero }}\right)$, a concentração letal mediana a $50 \%$ dos peixes $\left(\mathrm{CL}_{50}\right)$ e a observação do efeito tóxico aparente no comportamento natatório dos peixes sob ação do herbicida comercial e dos componentes puros diuron e hexazinona.

\section{MATERIAL E MÉTODOS}

Os ensaios de toxicidade aguda foram realizados no Laboratório de Recursos Hídricos da Universidade de Ribeirão Preto (UNAERP), conforme metodologia da ABNT (2004) e USEPA (2002), usando-se o Danio rerio como organismo-teste. Peixe de águas tropicais, conhecido como paulistinha ou zebrafish, chega a medir cinco centímetros de comprimento quando adulto. Apresenta coloração geral prateada, com cinco faixas longitudinais azul escuro e dimorfismo sexual. O macho é levemente dourado nas regiões abdominal, das nadadeiras peitorais e caudal, tendo o corpo mais alongado e delgado que a fêmea (STORER et al., 2000).

Os peixes paulistinhas (Danio rerio) de idade juvenil foram adquiridos em piscicultura para comércio de peixes ornamentais, cujos peixes são cultivados em tanques escavados no solo.

Após serem trazidos ao laboratório, os peixes foram aclimatados em reservatório de $200 \mathrm{~L}$ preenchido com água da rede de abastecimento desclorada com adição de tiossulfato de sódio. Efetuou-se renovação semanal de $30 \%$ do volume de água e os peixes permaneceram em observação durante 10 dias.

Preparou-se a água de diluição a partir da dissolução de sais em água deionizada. Para o preparo da água de diluição foram produzidas diferentes soluções estoque, sendo a solução denominada A composta de $1,5 \mathrm{mg}$ de sulfato de cálcio $\left(\mathrm{CaSO}_{4} \cdot 2 \mathrm{H}_{2} \mathrm{O}\right)$ por litro de água deionizada. A solução mista, denominada $\mathrm{B}$, continha $20,0 \mathrm{mg}$ de cloreto de potássio $(\mathrm{KCl}), 4800,0 \mathrm{mg}$ de bicarbonato de sódio $\left(\mathrm{NaHCO}_{3}\right)$ e $6100,0 \mathrm{mg}$ de sulfato de magnésio $\left(\mathrm{MgSO}_{4} \cdot 7 \mathrm{H}_{2} \mathrm{O}\right)$ dissolvidos em $1 \mathrm{~L}$ de água deionizada. A partir dessas duas soluções estoque preparou-se a água de diluição, sendo 2 litros da solução $A$ e 1 litro da solução $B$ dissolvidos em $100 \mathrm{~L}$ de água destilada e desclorada. A dureza total e o $\mathrm{pH}$ foram observados e anotados, corrigindo-se o $\mathrm{pH}$ quando necessário com solução de $\mathrm{HCl} 2 \mathrm{~N}$. Manteve-se o $\mathrm{pH}$ entre 7,0 e 7,6 e a dureza total em torno 40 e $48 \mathrm{mg}$ de $\mathrm{CaCO}_{3} \cdot \mathrm{L}^{-1}$.

Logo após o preparo da água, efetuou-se aeração durante 24 horas com auxílio de compressor de ar para alcançar a saturação de oxigênio, estabilização e dissolução total dos sais. Após o período de observação de 10 dias no reservatório, os peixes foram transferidos para aquário visando aclimatação de forma similar em $100 \%$ de água de diluição.

Os ensaios foram realizados em recipiente com 8 compartimentos de 5 litros e um compartimento posterior para equilíbrio de temperatura (Figuras 1 e 2), sendo utilizados 5 peixes por compartimento.

As concentrações aplicadas do herbicida comercial foram de 20, 30, 50, 60, 70, 80, 100, 300, $500,1000,1500,2000,2500,3000$ e $4000 \mathrm{mg}^{-\mathrm{L}^{-1}}$ dissolvidos em $3 \mathrm{~L}$ de água de diluição, previamente preparada. O compartimento, denominado de controle, foi mantido sem contaminação somente com água de diluição. Os parâmetros de controle da água foram: $\mathrm{pH}$, dureza, condutividade, oxigênio dissolvido e temperatura (APHA/AWWA/WEF, 2005).

Em cada concentração de água contaminada (em cada compartimento) foram colocados 5 peixes, inclusive no recipiente controle. A alimentação dos peixes foi suspensa 24 horas antes do início dos ensaios, sendo observada a mortalidade e o comportamento dos peixes após 24 e 48 horas.

A partir dos resultados dos ensaios preliminares estabeleceu-se o intervalo entre a menor concentração que não causou letalidade e a maior concentração que causou $100 \%$ de letalidade. Todos os ensaios seguiram o mesmo procedimento e em todos os casos não apresentaram letalidade no compartimento de controle. 


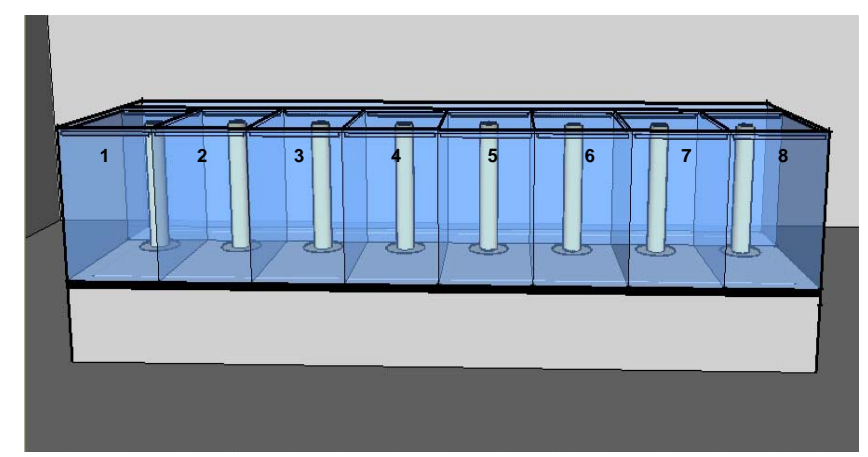

\section{FIGURA 1 - VISTA FRONTAL DO RECIPIENTE DE ENSAIO COM OS 8 COMPARTIMENTOS ANTERIORES}

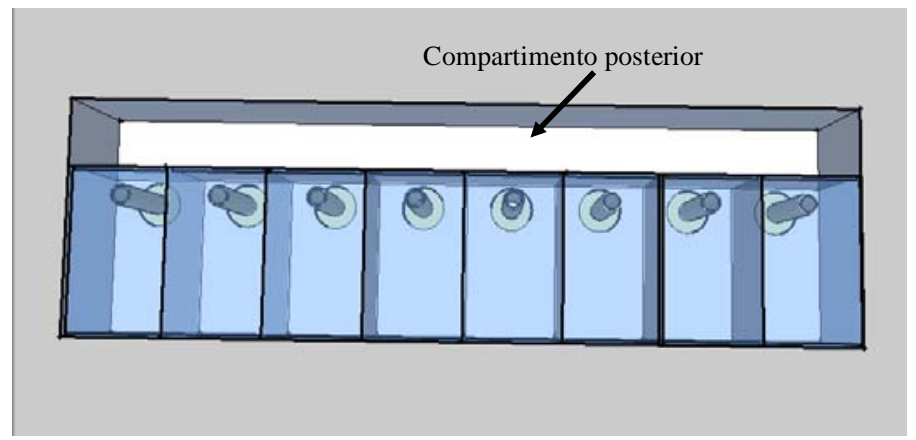

\section{FIGURA 2 - VISTA SUPERIOR DO RECIPIENTE DE ENSAIO COM OS 8 COMPARTIMENTOS ANTERIORES E O COMPARTIMENTO POSTERIOR}

Com base nos resultados obtidos nos ensaios preliminares determinaram-se as seguintes dosagens de herbicida comercial, 500; 550; 650; 700; 750; 800; 850 e 900 mg..-1 mais o controle, em duplicatas, sendo utilizados 10 peixes por recipiente.

Adotou-se o modelo Trimmed Spearman-Karber (HAMILTON, RUSSO e THURTON, 1977) no tratamento estatístico dos resultados para determinação da $\mathrm{CL}_{50}$, conforme recomendação da USEPA (2002). Posteriormente, foram realizados ensaios com os componentes ativos do herbicida comercial, diuron e hexazinona puros, cujas concentrações foram determinadas com base nas utilizadas no ensaio com o herbicida comercial.

Para os ensaios com hexazinona foram utilizadas as concentrações $0,66,73,86,92,99$, 106, 112 e $119 \mathrm{mg} \cdot \mathrm{L}^{-1}$. A baixa solubilidade do diuron em água (42 mg. $\left.\mathrm{L}^{-1}\right)$ não permitiu o uso das concentrações obtidas no ensaio definitivo com o herbicida comercial, sendo adotadas nos ensaios definitivos com diuron as concentrações de 0, 1, 5, 10, 20, 30, $40 \mathrm{mg} \cdot \mathrm{L}^{-1}$.

Conforme recomendações da USEPA (2002) realizou-se ensaio de sensibilidade com o organismoteste Danio rerio, utilizando como referência substância pura de dicromato de potássio $\left(\mathrm{K}_{2} \mathrm{Cr}_{2} \mathrm{O}_{7}\right)$ e a mesma metodologia empregada para o herbicida comercial. As concentrações utilizadas no ensaio de

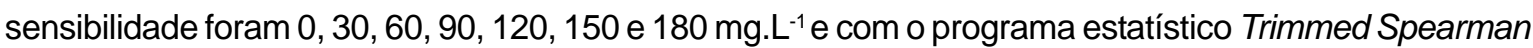
Karber obteve-se a concentração letal mediana $\left(\mathrm{CL}_{50}\right)$. Com base nesses resultados realizou-se ensaio com 16 réplicas, aplicando-se a concentração da $\mathrm{CL}_{50}$ de dicromato de potássio $\left(\mathrm{K}_{2} \mathrm{Cr}_{2} \mathrm{O}_{7}\right)$ em todos os recipientes.

Para o ensaio de repetibilidade com o herbicida comercial utilizou-se a mesma concentração obtida a partir do cálculo da $\mathrm{CL}_{50}$ para o herbicida comercial em 10 réplicas, seguindo a mesma metodologia. 
Nos cálculos estatísticos dos resultados dos ensaios de sensibilidade e repetibilidade usouse o programa aplicativo computacional Exce ${ }^{\circledR}$ (MICROSOFT, 2003). A partir desses resultados obtevese a média amostral (X), o limite superior de controle (LSC), o limite inferior de controle (LIC) e coeficiente de variação $(\mathrm{CV})$ que foi utilizado na construção das cartas de controle de sensibilidade e de repetibilidade.

\section{RESULTADOS E DISCUSSÃO}

Os resultados dos parâmetros de controle pH e dureza total das águas de diluição preparadas para a realização dos testes foram analisados e todos os valores obtidos mostraram-se de acordo com a norma 15088 da ABNT (2004), ou seja, pH da água de diluição entre 7,0 e 7,6 e dureza total de 40 a $48 \mathrm{mg}$ de $\mathrm{CaCO}_{3} \cdot \mathrm{L}^{-1}$.

Com os resultados do ensaio preliminar com o herbicida comercial estabeleceu-se o intervalo entre a menor concentração que causou $100 \%$ de mortalidade, $1000 \mathrm{mg} \cdot \mathrm{L}^{-1} \mathrm{e}$ a concentração efetiva zero $\left(\mathrm{CE}_{\text {zero }}\right) 400 \mathrm{mg} . \mathrm{L}^{-1} \mathrm{em}$ que não se observou mortalidade. A partir desses resultados determinouse o intervalo para o ensaio definitivo.

As variações de temperatura durante as medições foram pouco significativas no decorrer dos ensaios preliminares e definitivos (Figura 3). A temperatura mínima registrada foi de $25,5^{\circ} \mathrm{C}$ e a máxima de $26,5^{\circ} \mathrm{C}$, enquadrando-se na faixa aceitável de $26 \pm 1^{\circ} \mathrm{C}$ estabelecida pela ABNT (2004).

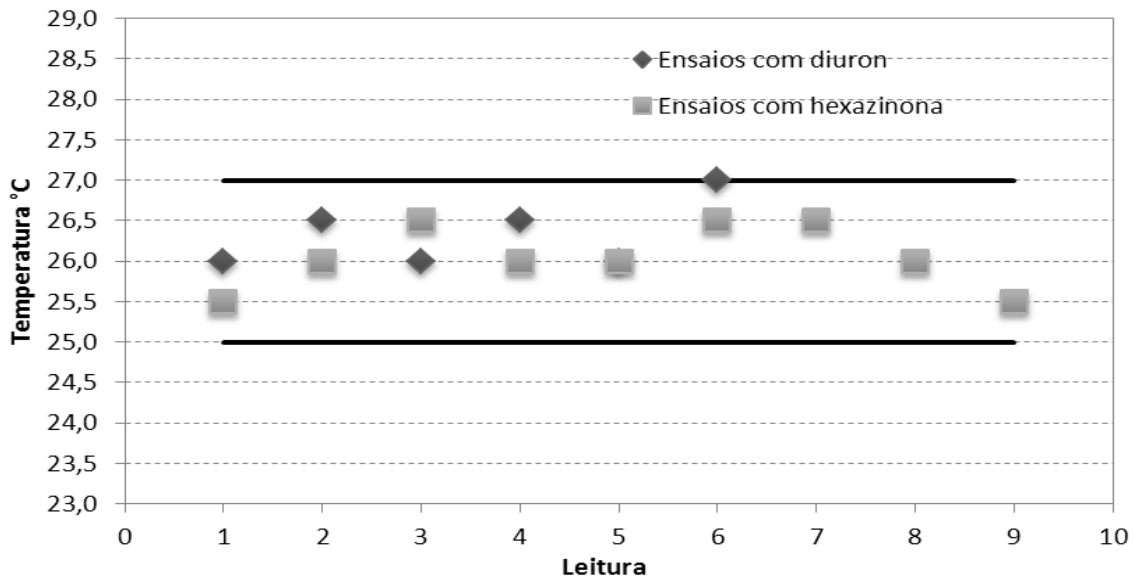

\section{FIGURA 3- CONTROLE DA TEMPERATURA DURANTE OS ENSAIOS PRELIMINARES E DEFINITIVOS COM O HERBICIDA COMERCIAL}

Com base nos resultados obtidos nos ensaios de ecotoxicidade aguda do herbicida comercial para o organismo-teste Danio rerio determinou-se a concentração letal mediana $\mathrm{CL}_{50-48 \mathrm{~h}}$ de $581,50 \mathrm{mg} . \mathrm{L}^{-1}$. Após 24 horas do início do ensaio foi observada maior incidência de mortalidade (Tabela 1).

Os resultados de controle da água em relação ao parâmetro dureza total apresentaram pequenas variações, aumentando ligeiramente quando comparado à dureza total encontrada nos recipientes de controle, indicando que os demais componentes do herbicida comercial nas concentrações estudadas causaram tais variações. As alterações de $\mathrm{pH}$ foram observadas principalmente nos recipientes de ensaio com o herbicida comercial, devendo-se a ligeira queda do $\mathrm{pH}$ às excretas metabólicas produzidas pelos organismos-testes e pela degradação da matéria orgânica. Na Tabela 2 são apresentadas as concentrações dosadas do herbicida comercial e os resultados obtidos no controle da água de diluição no início e após 48 horas. 

TOTAL DE MORTES EM 24h E 48h E A PORCENTAGEM TOTAL DE MORTOS

\begin{tabular}{|c|c|c|c|c|}
\hline \multirow{2}{*}{$\begin{array}{c}\text { Concentraçãa do herpicida } \\
\left.\text { comercial (mg. } L^{-}\right)\end{array}$} & \multirow{2}{*}{$N^{\circ}$ de peixes } & \multicolumn{2}{|c|}{$N^{\circ}$ total de mortes } & \multirow{2}{*}{$\begin{array}{c}\text { Mortalidade } \\
(\%)\end{array}$} \\
\hline & & $24 \mathrm{~h}$ & $48 \mathrm{~h}$ & \\
\hline Controle & 20 & 0 & 0 & 0 \\
\hline 500 & 20 & 1 & 8 & 45 \\
\hline 550 & 20 & 1 & 7 & 40 \\
\hline 650 & 20 & 1 & 12 & 65 \\
\hline 700 & 20 & 2 & 16 & 90 \\
\hline 750 & 20 & 7 & 11 & 90 \\
\hline 800 & 20 & 3 & 16 & 95 \\
\hline 850 & 20 & 7 & 13 & 100 \\
\hline 900 & 20 & 8 & 12 & 100 \\
\hline
\end{tabular}

TABELA 2 - RESULTADOS DE VALORES MÉDIOS DOS PARÂMETROS DE CONTROLE DOS ENSAIOS COM Danio rerio PARA DIFERENTES CONCENTRAÇÕES DO HERBICIDA COMERCIAL

\begin{tabular}{ccccccccc}
\hline $\begin{array}{c}\text { Herbicida } \\
\text { comercial } \\
\text { mg/L }\end{array}$ & $\mathrm{pH}$ & $\begin{array}{c}\text { Dureza } \\
\mathrm{mgCaCO}_{3} / \mathrm{L}\end{array}$ & $\begin{array}{c}\mathrm{OD} \\
\mathrm{mg} \mathrm{O}_{2} / \mathrm{L}\end{array}$ & $\begin{array}{c}\text { Cond. } \\
\mu \mathrm{S} / \mathrm{cm}\end{array}$ & $\mathrm{pH}$ & $\begin{array}{c}\text { 4ureza horas } \\
\mathrm{mgCaCO}_{3} / \mathrm{L}\end{array}$ & $\begin{array}{c}\mathrm{OD} \\
\mathrm{mgO}_{2} / \mathrm{L}\end{array}$ & $\begin{array}{c}\text { Cond. } \\
\mu \mathrm{Scm}\end{array}$ \\
\hline Controle 1 & 7,4 & 42 & 7,2 & 147 & 7,3 & 42 & 5,7 & 142 \\
500 & 7,5 & 45 & 7,1 & 163 & 7,2 & 45 & 5,8 & 161 \\
550 & 7,4 & 45 & 7,2 & 165 & 7,2 & 45 & 4,7 & 163 \\
Controle 2 & 7,6 & 44 & 7,1 & 151 & 7,5 & 44 & 5,5 & 152 \\
650 & 7,7 & 47 & 7,1 & 166 & 7,3 & 48 & 5,3 & 167 \\
700 & 7,8 & 48 & 7,1 & 169 & 7,3 & 48 & 5,5 & 171 \\
750 & 7,8 & 48 & 7,1 & 171 & 7,2 & 49 & 5,6 & 174 \\
Controle 3 & 7,5 & 46 & 7,2 & 150 & 7,2 & 46 & 6,1 & 149 \\
800 & 7,7 & 48 & 7,2 & 171 & 7,3 & 48 & 6,2 & 174 \\
850 & 7,8 & 48 & 7,2 & 174 & 7,3 & 48 & 5,8 & 174 \\
900 & 7,8 & 49 & 7,2 & 177 & 7,3 & 49 & 5,8 & 174 \\
\hline
\end{tabular}

Nota: $\mathrm{OD}=$ oxigênio dissolvido; Cond = Condutividade elétrica.

A crescente elevação das concentrações do herbicida comercial refletiu-se no aumento da condutividade, embora a condutividade inicial não tenha apresentado alterações significativas em relação à condutividade final. A redução na concentração do oxigênio dissolvido pode ser atribuída à degradação de matéria orgânica e aumento do consumo de oxigênio pelos peixes que se encontravam sob situação de estresse, mas sempre mantida acima do limite mínimo permitido (4 $\left.\mathrm{mg}^{-\mathrm{L}^{-1}}\right)$ pela ABNT (2004).

No início do ensaio com o herbicida comercial nas concentrações entre 40 e $50 \mathrm{mg} \cdot \mathrm{L}^{-1}$ observouse hiperatividade dos peixes relacionada ao aumento do movimento natatório em mais de $50 \%$ dos organismos. $\mathrm{O}$ aumento da atividade locomotora nesse caso está relacionada à resposta de escape.

Em concentrações acima de 50 mg. $\mathrm{L}^{-1}$ observou-se hipoatividade em mais de $50 \%$ dos organismos. A diminuição do movimento natatório pode ser explicada como comportamento adaptativo ao estresse devido à possível realocação de energia para outros processos fisiológicos, resultando em menor quantidade de energia para a locomoção. 
Nos ensaios de sensibilidade utilizando como substância de referência o dicromato de potássio $\left(\mathrm{K}_{2} \mathrm{Cr}_{2} \mathrm{O}_{7}\right)$, inicialmente obteve-se a concentração letal mediana $\mathrm{CL}_{50}$ de aproximadamente $114 \mathrm{mg} \cdot \mathrm{L}^{-1}$. A partir das réplicas realizadas com a $\mathrm{CL}_{50}$ foram obtidos dados para os cálculos, cujo desvio-padrão foi de $\pm 2,17$ e o coeficiente de variação de $20 \%$. Esse resultado enquadra-se no limite do coeficiente de variação (CV) de $45 \%$ proposto pela USEPA (2002). Na Figura 4 estão descritos os resultados da carta-controle de sensibilidade, sendo a média amostral (X) estabelecida em 5,5, o limite superior de controle (LSC) em 7,7 e o limite inferior (LIC) em 3,3.

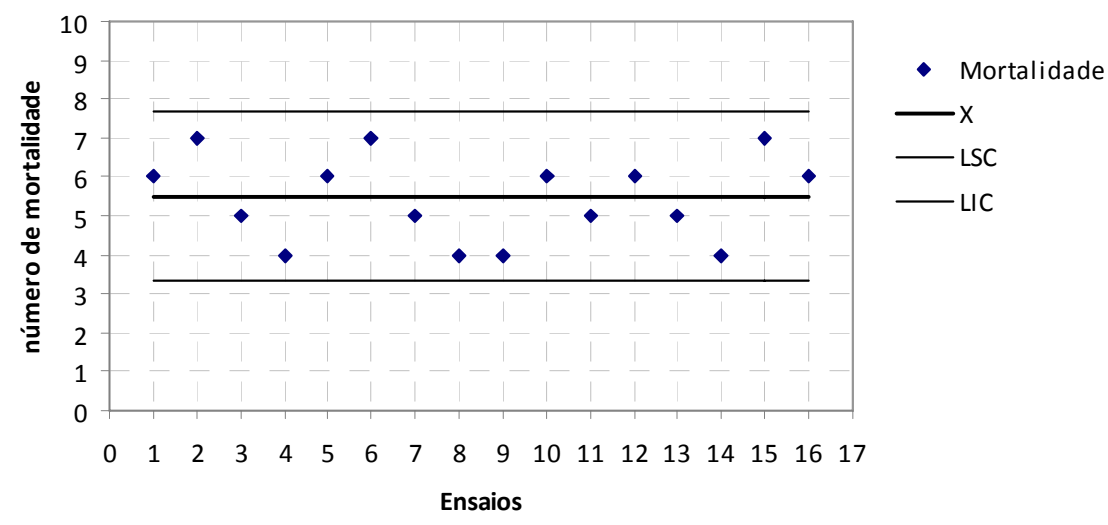

\section{FIGURA 4 - CARTA-CONTROLE DE SENSIBILIDADE À SUBSTÂNCIA DE REFERÊNCIA DICROMATO DE POTÁSSIO $\left(\mathrm{K}_{2} \mathrm{CR}_{2} \mathrm{O}_{7}\right)$, COM OS RESULTADOS DAS MORTALIDADES, A MÉDIA AMOSTRAL (X), O LIMITE SUPERIOR DE CONTROLE (LSC) E O LIMITE INFERIOR DE CONTROLE (LIC)}

No ensaio de repetibilidade realizado com o herbicida comercial obteve-se desvio-padrão de $\pm 2,53$ e coeficiente de variação $(C V)$ de $21 \%$, enquadrando no limite do coeficiente de variação de $45 \%$ proposto pela USEPA (2002). Na Figura 5 estão apresentados os resultados do ensaio de repetibilidade com o herbicida comercial, cuja média amostral $(X)$ ficou em 6 , o limite superior de controle (LSC) em 8,5 e o limite inferior (LIC) em 3,5.

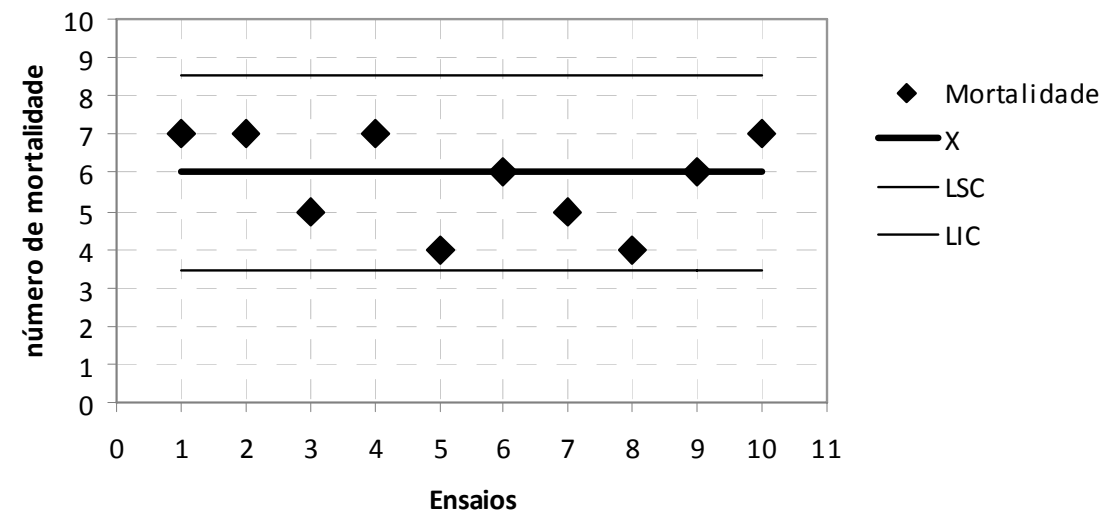

FIGURA 5 - RESULTADOS DAS MORTALIDADES DO ENSAIO DE REPETIBILIDADE COM O HERBICIDA COMERCIAL, MÉDIA AMOSTRAL (X), LIMITE SUPERIOR DE CONTROLE (LSC) E LIMITE INFERIOR DE CONTROLE (LIC)

A variação de temperatura dos ensaios com hexazinona e com o diuron manteve-se na faixa aceitável $\left(26 \pm 1^{\circ} \mathrm{C}\right)$ pela ABNT (2004), entre a máxima de $27,0^{\circ} \mathrm{C}$ e a mínima de $25,5^{\circ} \mathrm{C}$ (Figura 6 ). 


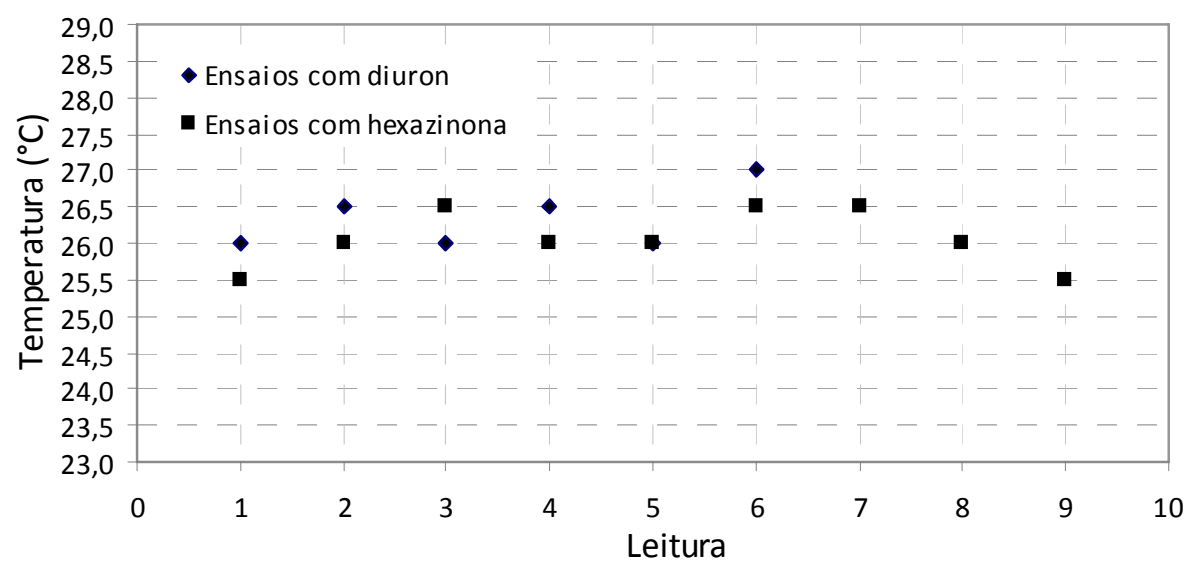

\section{FIGURA 6 - CONTROLE DA TEMPERATURA DURANTE OS ENSAIOS DEFINITIVOS COM DIURON E HEXAZINONA}

Os resultados do parâmetro de dureza total obtidos durante o ensaio definitivo com hexazinona não apresentaram alteração significativa, assim como as variações de $\mathrm{pH}$ foram pouco significativas. As concentrações de hexazinona utilizadas não foram suficientes para causar alterações significativas na condutividade durante os ensaios. Verificou-se diminuição na concentração de oxigênio dissolvido, no entanto os níveis de oxigênio mantiveram-se acima do limite mínimo de $4 \mathrm{mg} . \mathrm{L}^{-1}$. Os resultados dos valores médios obtidos no controle do ensaio com hexazinona estão apresentados na Tabela 3.

\section{TABELA 3 - RESULTADOS DOS VALORES MÉDIOS DOS PARÂMETROS DE CONTROLE OBTIDOS NOS ENSAIOS COM Danio rerio PARA DIFERENTES CONCENTRAÇÕES DE HEXAZINONA}

\begin{tabular}{|c|c|c|c|c|c|c|c|c|}
\hline \multirow[b]{2}{*}{$\begin{array}{l}\text { Hexazinona } \\
\mathrm{mg} / \mathrm{L}\end{array}$} & \multicolumn{4}{|c|}{ Início } & \multicolumn{4}{|c|}{48 horas } \\
\hline & $\mathrm{pH}$ & $\begin{array}{l}\text { Dureza total } \\
\mathrm{mg} \mathrm{CaCO}_{3} / \mathrm{L}\end{array}$ & $\begin{array}{l}\text { OD } \\
\mathrm{mg} / \mathrm{L}\end{array}$ & $\begin{array}{l}\text { Cond. } \\
\mu S / \mathrm{cm}\end{array}$ & $\mathrm{pH}$ & $\begin{array}{l}\text { Dureza total } \\
\mathrm{mg} \mathrm{CaCO}_{3} / \mathrm{L}\end{array}$ & $\begin{array}{l}\text { OD } \\
\mathrm{mg} / \mathrm{L}\end{array}$ & $\begin{array}{l}\text { Cond. } \\
\mu S / \mathrm{cm}\end{array}$ \\
\hline Controle 1 & 7,2 & 43 & 7,3 & 141 & 7,4 & 43 & 6,4 & 141 \\
\hline 66 & 7,2 & 42 & 7,3 & 140 & 7,5 & 43 & 6,1 & 140 \\
\hline 73 & 7,2 & 42 & 7,3 & 140 & 7,5 & 41 & 6,2 & 141 \\
\hline Controle 2 & 7,4 & 41 & 7,4 & 130 & 7,3 & 41 & 6,6 & 132 \\
\hline 86 & 7,4 & 41 & 7,3 & 131 & 7,4 & 41 & 6,4 & 131 \\
\hline 92 & 7,4 & 41 & 7,3 & 131 & 7,4 & 41 & 6,1 & 132 \\
\hline 99 & 7,4 & 41 & 7,2 & 131 & 7,3 & 41 & 6,3 & 132 \\
\hline Controle 3 & 7,3 & 41 & 7,2 & 134 & 7,3 & 41 & 6,4 & 132 \\
\hline 106 & 7,3 & 42 & 7,3 & 135 & 7,3 & 42 & 6,5 & 131 \\
\hline 112 & 73 & 41 & 7,3 & 135 & 7,3 & 41 & 6,2 & 131 \\
\hline 119 & 7,1 & 41 & 7,3 & 135 & 7,1 & 49 & 6,0 & 131 \\
\hline
\end{tabular}

Nota: $\mathrm{OD}=$ oxigênio dissolvido; Cond = Condutividade elétrica.

Nos ensaios realizados com diuron, os valores da dureza total analisados não evidenciaram alterações significativas e o pH manteve-se estável. As concentrações de diuron utilizadas não foram suficientes para causar alterações significativas na condutividade durante os ensaios. Os resultados 
dos valores médios dos parâmetros de controle obtidos durante os ensaios com o herbicida diuron estão apresentados na Tabela 4.

\section{TABELA 4 - RESULTADOS DOS VALORES MÉDIOS DOS PARÂMETROS DE CONTROLE OBTIDOS NOS ENSAIOS COM Danio rerio PARA DIFERENTES CONCENTRAÇÕES DE DIURON}

\begin{tabular}{|c|c|c|c|c|c|c|c|c|}
\hline \multirow[b]{2}{*}{$\begin{array}{l}\text { Hexazinona } \\
\mathrm{mg} / \mathrm{L}\end{array}$} & \multicolumn{4}{|c|}{ Início } & \multicolumn{4}{|c|}{48 horas } \\
\hline & $\mathrm{pH}$ & $\begin{array}{l}\text { Dureza total } \\
\mathrm{mg} \mathrm{CaCO}_{3} / \mathrm{L}\end{array}$ & $\begin{array}{l}\text { OD } \\
\mathrm{mg} / \mathrm{L}\end{array}$ & $\begin{array}{l}\text { Cond. } \\
\mu \mathrm{S} / \mathrm{cm}\end{array}$ & $\mathrm{pH}$ & $\begin{array}{l}\text { Dureza total } \\
\mathrm{mg} \mathrm{CaCO}_{3} / \mathrm{L}\end{array}$ & $\begin{array}{l}\text { OD } \\
\mathrm{mg} / \mathrm{L}\end{array}$ & $\begin{array}{l}\text { Cond. } \\
\mu S / c m\end{array}$ \\
\hline Controle 1 & 7,2 & 43 & 7,3 & 141 & 7,4 & 43 & 6,4 & 141 \\
\hline 66 & 7,2 & 42 & 7,3 & 140 & 7,5 & 43 & 6,1 & 140 \\
\hline 73 & 7,2 & 42 & 7,3 & 140 & 7,5 & 41 & 6,2 & 141 \\
\hline Controle 2 & 7,4 & 41 & 7,4 & 130 & 7,3 & 41 & 6,6 & 132 \\
\hline 86 & 7,4 & 41 & 7,3 & 131 & 7,4 & 41 & 6,4 & 131 \\
\hline 92 & 7,4 & 41 & 7,3 & 131 & 7,4 & 41 & 6,1 & 132 \\
\hline 99 & 7,4 & 41 & 7,2 & 131 & 7,3 & 41 & 6,3 & 132 \\
\hline Controle 3 & 7,3 & 41 & 7,2 & 134 & 7,3 & 41 & 6,4 & 132 \\
\hline 106 & 7,3 & 42 & 7,3 & 135 & 7,3 & 42 & 6,5 & 131 \\
\hline 112 & 73 & 41 & 7,3 & 135 & 7,3 & 41 & 6,2 & 131 \\
\hline 119 & 7,1 & 41 & 7,3 & 135 & 7,1 & 49 & 6,0 & 131 \\
\hline
\end{tabular}

Nota: OD = oxigênio dissolvido; Cond = Condutividade elétrica.

Durante os ensaios com o diuron houve diminuição na concentração do oxigênio dissolvido, no entanto os níveis de oxigênio se mantiveram acima do limite mínimo de 4 mg.L-1.

Os ensaios realizados tanto com o herbicida diuron quanto com o herbicida hexazinona, nas concentrações que foram testadas, obtidas a partir do ensaio definitivo com o herbicida comercial não apresentaram mortalidade e aparentemente não afetaram o comportamento natatório dos peixes.

\section{CONCLUSÃO}

Nas condições em que foram realizados os ensaios, o herbicida comercial estudado apresentou $\mathrm{CL}_{50}$ de 581,5 mg.L-1 para o organismo-teste Danio rerio.

Os organismos revelaram alterações significativas no comportamento em concentrações do herbicida comercial entre 40 e $50 \mathrm{mg} \cdot \mathrm{L}^{-1}$ e em concentrações acima de $50 \mathrm{mg} \cdot \mathrm{L}^{-1}$, demonstrando que nessas concentrações são observadas modificações que podem vir a causar impactos ambientais negativos em nicho ecológico que essa espécie de organismo possa vir a representar.

Para os principais compostos do herbicida comercial estudados, separadamente, não foi observada mortalidade ou alteração comportamental nos organismos-teste nas concentrações utilizadas.

Os resultados obtidos nos ensaios de sensibilidade realizados com a substância de referência dicromato de potássio apresentaram coeficiente de variação abaixo de $45 \%$. Considerando esse coeficiente, concluiu-se que o ensaio de repetibilidade realizado para a validação dos resultados com o herbicida comercial foi satisfatório.

\section{ABSTRACT \\ ACUTE TOXICITY OF HERBICIDES DIURON AND HEXAZINONE TO Danio rerio}

The objective of this study was to evaluate the toxicity of a commercial herbicide extensively used in sugarcane 
cultivation, composed of diuron, hexazinone and inert ingredients using Danio rerio as test organism. Laboratory tests were performed according to the recommendations of the ABNT 15088 (2004) and USEPA (2002) standards. The water control parameters were $\mathrm{pH}$, total water hardness, electrical conductivity, dissolved oxygen and temperature, swimming activity and mortality of the test organism. The lethal concentration $\left(\mathrm{LC}_{50}\right.$ ) of the commercial herbicide for Danio rerio was of $581.50 \mathrm{mg} \cdot \mathrm{L}^{-1}$. Significant behavioral changes were observed at concentrations starting from $50 \mathrm{mg} \cdot \mathrm{L}^{-1}$ of the commercial herbicide, causing hypoactivity of swimming movements in over $50 \%$ of the test organisms. To evaluate the toxicity of the inert ingredients, which account for $40 \% \mathrm{p} / \mathrm{p}$ of the herbicide's composition, the compounds hexazinone and diuron were tested separately. At the studied concentrations, hexazinone and diuron did not cause mortality or altered swimming movements. It was concluded that the inert ingredients in the composition of the commercial herbicide were the major cause for acute toxicity to Danio rerio.

\section{KEY-WORDS: SUGARCANE; ECOTOXICOLOGY; Danio rerio; DIURON; HEXAZINONA.}

\section{REFERÊNCIAS}

1 ABNT. Associação Brasileira de Normas Técnicas. NBR 15088: ecotoxicologia aquática - toxicidade aguda - método de ensaio em peixes. São Paulo, 2004. 19 p.

2 APHA, AWWA, WEF. American Public Health Association; American Water Works Association; Water Environment Federation. Standard methods for the examination of the water and wastewater. $21^{\text {st }}$. Washington, D.C, 2005.

3 BAGANZ, D. Comparing locomotor behaviour of fish species Danio rerio and Leucaspius delineatus under the influence of chemical stressors. 2005. 212 f. (Ph.D. Thesis), Mathematisch-Naturwissenschaftlichen Fakultät I, der Humboldt-Universität zu Berlin. Berlin, 2005.

4 BRASIL. Conselho Nacional do Meio Ambiente. Resolução $n^{\circ}$ 357, de 31 de março de 2005. Classificação dos corpos de água e diretrizes ambientais para o seu enquadramento, bem como estabelece as condições e padrões de lançamento de efluentes, e dá outras providências. Diário Oficial [da] República Federativa do Brasil Brasília, DF, 2005.

5 BRASIL. Ministério da Saúde. Portaria $\mathrm{n}^{\circ} 518$ GM de 25 de março de 2004. Estabelece os procedimentos e responsabilidades relativos ao controle e vigilância da qualidade da água para consumo humano e seu padrão de potabilidade, e dá outras providências, Diário Oficial [da] República Federativa do Brasil, Brasília, DF, 2004.

6 DANTAS, A.D.B.; PASCHOALATO, C.F.P.R.; BALLEJO, R.R.; Di BERNARDO, L. Pré-oxidação e adsorção em carvão ativado granular para remoção dos herbicidas diuron e hexazinona de água subterrânea. Eng. Sanit. Ambient., Rio de Janeiro, v. 14, n. 3, p373-380, jul/set. 2009.

7 FERRÃO FILHO, A. S.; CUNHA, R.; MAGALHÃES, V.F.; SOARES, M.C.S.; BAPTISTA, D.F. Evaluation of sub-lethal toxicity of cyanobacteria on the swimming activity of aquatic organisms by image analysis. J. Braz. Soc. Ecotoxicol, v. 2 , n. 2 , p. $93-100,2007$

8 FRELLO, C.P. Avaliação da toxicidade aguda do pesticide carbofuran utilizando reativos biológicos Poecilla reticulata e Daphnia magna. 96 p. Dissertação (Mestrado em Engenharia Ambiental), Universidade Federal de Santa Catarina, Florianópolis, SC, 1998.

9 HAMILTON, M.A. RUSSO, R.C.; THURTON, R.V. Trimed Spearman - Karber method for estimating median lethal concentrations in toxicity bioassays. Environmental Science and Technology, New York, v. 11, n. 7, p. 714-719, 1977.

10 HELFRICH, L.A.; WEIGMANN, D.L.; HIPKINS, P.; SPINSON, E.R. Pesticides and aquatic animals: a guide to reducing impacts on aquatics systems. 1996. Disponível em: www.ext.vt.edu/pubs/waterquality/420-013/420-013.pdf. Acesso em: 05/05/2009.

11 HOLTH, T.F.; NOURIZADEH-LILLABADI, R.; BLAESBJERG, M.; GRUNG, M.; HOLBECH, H.; PETERSEN, G.l.; ALESTROM, P.; HYLLAND, K. Differential gene expression and biomarkers in zebrafish (Danio rerio) following exposure to produced water components. Aquatic Toxicology, v. 90, p. 277-291, Dec. 2008.

12 IEA. Instituto de Economia Agrícola. Cana-de-açúcar para indústria: o quanto vai precisar crescer. Análise e Indicadores do Agronegócio, v.1, n.10, p. 92-99, 2006.

13 JONSSON, C.M.; MAIA, A.H.N.Toxicidade aguda do herbicida clomazone no peixe hyphessobrycon - scholzei: avaliação da concentração letal mediana e de alterações no conteúdo de nutrientes. Pesticidas: revista de ecotoxicologia e meio ambiente, Curitiba, v. 8, p. 101-110, 1998.

14 LANÇAS, F.M. Estudo de resíduo de diuron e hexazinona em água superficial (de Rio) no Brasil. São Carlos: Laboratório de Cromatografia/Instituto de Química de São Carlos/USP, 2002. (RE-98-053 A.02). 
15 LOMBARDI, J.V. Fundamentos de toxicologia aquática. In: RANZANI-PAIVA, M.J.T.; TAKEMOTO, R.M.; LIZAMA, M. DE LOS A.P. Sanidade de organismos aquáticos. São Paulo: Varela, 2004. p. 263-272.

16 MATALLO, M.; LUCHINI, L.; GOMES, M.; SPADOTTO, C.; CERDEIRA, A.; MARIN, G. Lixiviação dos herbicidas tebutiuron e diuron em colunas de solo. Pesticidas: revista de ecotoxicologia e meio ambiente, Curitiba, v. 13, p. 83-90, jan./dez. 2003.

17 MICROSOFT Office Professional Excel Version 2003. Seatle: Microsoft Coporation, 2003. CD-ROM.

18 NAKAGOME, F.; NOLDIN, J.; RESGALLA JR., C. Toxicidade aguda de alguns herbicidas e inseticidas utilizados em lavouras de arroz irrigado sobre o peixe Danio rerio. Pesticidas: revista de ecotoxicologia e meio ambiente, Curitiba, v.17, p.117-122, jan./dez. 2007.

19 PÁDUA, V. L. Remoção de microcontaminantes orgânicos no tratamento de água para consumo humano. Rio de Janeiro: ABES, 2009. 392 p. (Projeto PROSAB).

20 PASCHOALATO, C.F.P.R.; DANTAS, A.D.B.; ROSA, I.D.A.; FALEIROS, R.D.J.R.; BERNARDO, Luiz Di. Uso de carvão ativado para remoção dos herbicidas diuron e hexazinona de água. Revista DAE, São Paulo, v. 179, p. 34-41, 2009.

21 PESSOA, M.; GOMES, M.; NEVES, M.; CERDEIRA, A.; DE SOUZA, M. Identificação de áreas de exposição ao risco de contaminação de águas subterrâneas pelos herbicidas atrazina, diuron e tebutiuron. Pesticidas: revista de ecotoxicologia e meio ambiente, Curitiba, v.13, p. 111-122, jan./dez. 2003.

22 PESSOA, M.C.P.Y.; SCRAMIN, S.; CHAIN, A.; FERRACCINI, V.L. Avaliação do potencial de transporte de agrotóxicos usados no Brasil por modelos screening e planilha eletrônica. Jaguariúna: Embrapa Meio Ambiente, 2007. 24 p. (Embrapa Meio Ambiente. Boletim de Pesquisa e Desenvolvimento, 44).

23 POLITANO, W.; PISSARRA, T.C.T. Avaliação por fotointerpretação das áreas de abrangência dos diferentes estados da erosão acelerada do solo em canaviais e pomares de citros. Eng. Agríc., Jaboticabal, v. 25, n. 1, p. 242-252, jan./abr.2005.

24 RESGALLA JR., C.; NOLDIN, J.A.; SATO, G.; EBERHARDT, D.S. Toxicidade aguda de herbicidas e inseticidas utilizados na cultura de arroz irrigado sobre juvenis de carpa (Cyprinus carpio). Pesticidas: revista de ecotoxicologia e meio ambiente, Curitiba, v. 12, p. 59-68, 2002.

25 STORER, T. I,; USINGER, R. L.; STEBBINS, R. C.; NYBAKKEN, J. W. Zoologia geral. 6. ed. São Paulo: Companhia Editora Nacional, 2000. $816 \mathrm{p}$.

26 UNICA. União da Indústria de Cana-de-Açúcar. Etanol de cana-de-açúcar. Disponível em: http://www. unica. com. br /content/ show.asp ?cntCode=9E97665F-3A81-46F2 BF69 -26E00C323988. Acesso em: 18/09/2008.

27 USEPA. United States Environmental Protection Agency. Methods for measuring the acute toxicity of efluents and receiving waters to freshwater and marine organisms. $5^{\text {th }}$ ed. Washignton, DC, 2002. 